\title{
Prevention of retrosternal adhesion by novel biocompatible glue derived from food additives
}

\author{
Tetsuya Kamitani, MD, ${ }^{\text {a }}$ Hidetoshi Masumoto, MD, PhD, ${ }^{a}$ Hirokazu Kotani, MD, PhD, ${ }^{\text {b }}$ \\ Tadashi Ikeda, MD, PhD, ${ }^{\mathrm{a}}$ Suong-Hyu Hyon, $\mathrm{PhD},{ }^{\mathrm{c}}$ and Ryuzo Sakata, MD, PhD ${ }^{\mathrm{a}}$
}

\begin{abstract}
Background: Postoperative retrosternal adhesion increases the risk of cardiac injury during cardiac reoperation. We created a novel biodegradable glue called "Lydex" that is derived from food additives. The purpose of this study is to evaluate this new biomaterial's biocompatibility and its preventive effect on retrosternal adhesion.

Methods: We performed a median sternotomy and anterior pericardiectomy on Japanese white rabbits, and then closed the chest (control, group 1; $\mathrm{n}=12$ ), implanted an expanded polytetrafluoroethylene membrane (group 2; $\mathrm{n}=12$ ), or applied Lydex (group 3; $\mathrm{n}=12$ ) before closure. After 4 weeks, we evaluated macroscopic adhesion (each group; $\mathrm{n}=6$ ) and microscopic findings for fibrosis and macrophage infiltration (each group; $\mathrm{n}=6$ ).
\end{abstract}

Results: In group 3 , the retrosternal adhesion score was significantly lower than in group $1(P=.0022)$. There was no significant difference between groups 2 and 3 . The fibrotic area ratio was significantly lower in group 3 than in groups 1 and $2(P<.001$ vs group $1 ; P<.001$ vs group 2$)$. In group 3 , the macrophage count was significantly lower than in group $2(P<.001)$ and almost equal to that in group 1.

Conclusions: Our findings indicate that Lydex reduces retrosternal adhesion and attenuates the progression of fibrosis with excellent biocompatibility. Lydex is a next-generation substance for safer cardiac reoperation, with excellent capability for preventing adhesion, biocompatible and biodegradable properties, and lower potential for viral infections related to human plasma or other animal-derived products. (J Thorac Cardiovasc Surg 2013;146:1232-8)

In the field of cardiac surgery, it is reported that reoperation is closely related to increased hospital mortality. ${ }^{1}$ Postoperative retrosternal adhesion increases the risk for cardiac injury and bleeding during cardiac reoperation, especially at the time of resternotomy. ${ }^{2,3}$ It is widely known that closure of the pericardium at the initial operation reduces the risk of injury on reoperation. However, there are situations in which surgeons cannot close the pericardium, such as coronary artery bypass grafting with internal thoracic artery grafts or operations performed in patients with severe heart failure. Furthermore, in pediatric cardiac surgery for congenital heart disease, patients may require repetitive reoperations in many palliative cases.

From the Department of Cardiovascular Surgery, ${ }^{\mathrm{a}}$ Kyoto University Graduate School of Medicine, Kyoto, Japan; Department of Forensic Medicine and Molecular Pathology, ${ }^{\mathrm{b}}$ Kyoto University Graduate School of Medicine, Kyoto, Japan; and Center for Fiber and Textile Science, ${ }^{\mathrm{c}}$ Kyoto Institute of Technology, Kyoto, Japan.

This work was supported by research grants from the Ministry of Education, Culture, Sports, Science, and Technology, Japan (to T.I.)

Disclosures: Dr Hyon holds a patent right in the United States, the People's Republic of China, and Japan for the biodegradable glue Lydex. He is one of the patent inventors. However, because the substance is not produced commercially, incomes (eg, a patent loyalty) have not been received. All other authors have nothing to disclose with regard to commercial support.

T.K. and H.M. equally contributed to this work.

Received for publication Aug 29, 2012; revisions received Jan 10, 2013; accepted for publication Feb 8, 2013; available ahead of print April 12, 2013.

Address for reprints: Tadashi Ikeda, MD, PhD, Department of Cardiovascular Surgery, Kyoto University Graduate School of Medicine, 54 Shogoin Kawaharacho, Sakyo-ku, Kyoto 603-8507, Japan (E-mail: ike@kuhp.kyoto-u.ac.jp).

0022-5223/\$36.00

Copyright (c) 2013 by The American Association for Thoracic Surgery

http://dx.doi.org/10.1016/j.jtcvs.2013.02.001
Surgeons have used a variety of biological or synthetic materials as a substitute for the pericardium to resolve retrosternal adhesion in such situations; these include expanded polytetrafluoroethylene (ePTFE) membranes, ${ }^{4}$ silicone rubbers, ${ }^{5}$ glutaraldehyde-treated bovine or porcine pericardium xenografts, ${ }^{6,7}$ and others. However, several disadvantages, such as dense adhesion or severe inflammation, have been reported with the use of pericardial substitutes. ${ }^{8}$ Furthermore, the application of nondegradable material as a pericardial substitute may increase the risk of mediastinitis, one of the critical complications after cardiac surgery, because of colonization of infective microorganisms on the material. ${ }^{9}$

Many researchers have reported that bioabsorbable membranes effectively prevented the formation of retrosternal adhesion in experimental studies. ${ }^{10-13}$ However, these membranes have never been widely used in the clinical setting, possibly because of insufficient clinical evidence on their beneficial effects or safety. Furthermore, materials made from human plasma or other animal-derived products may hold risks for viral infection.

We recently created a novel bioabsorbable glue called "Lydex," which is a newly developed chemical compound derived from the food additives dextran and $\varepsilon$-poly (L-lysine). ${ }^{14}$ Dextran is a polysaccharide that is widely used as a plasma volume expander and thickener in the clinical setting. $\varepsilon$-Poly (L-lysine) is a small natural homopolymer of the essential amino acid L-lysine and is used as an antibacterial agent. As food additives, dextrans are used as coating agents in food-contact surfaces, thickening agents, stabilizers, or 


\section{Abbreviation and Acronym \\ ePTFE $=$ expanded polytetrafluoroethylene}

bulking agents. $\varepsilon$-Polylysine has a history of use as a preservative in multiple foods, including several staples in the Asian diet, such as sushi or boiled rice. ${ }^{15}$ We have previously reported the effectiveness of the biodegradable glue as a novel surgical sealant for lung air leakage ${ }^{14}$ or an attachment medium for sustained release of antibiotics or antiarrhythmic drugs in experimental animal models. ${ }^{16-18}$ The purpose of this study is to examine the effect of the biodegradable glue on retrosternal adhesion and, using a rabbit pericardiectomy model, to evaluate the inflammatory and fibrogenic capacity and biocompatibility of the glue for wide clinical application of this new material in cardiac surgery.

\section{MATERIALS AND METHODS}

The study protocol was approved by the Kyoto University Ethics Committee for Animal Research. All animals received humane care in compliance with the guidelines prescribed in the Principles of Laboratory Animal Care, formulated by the National Society for Medical Research, and the Guide for the Care and Use of Laboratory Animals, created by the Institute of Laboratory Animal Resources of the National Research Council and published by the National Academy Press (revised 2011)

\section{New Absorbable Glue: Lydex}

The absorbable glue called "Lydex" is made of the plant-derived food additives dextran and 10\% ع-poly (L-lysine) solution; both are approved by the US Food and Drug Administration. The production process of the biodegradable glue has been described in detail. ${ }^{14}$ In brief, dextran was dissolved in distilled water. Sodium periodate was dissolved in water, and this solution was added to the previously mentioned dextran solution. The aldehyde groups were introduced by periodate oxidation of dextran (Malaprade oxidation). Recovered aldehyde dextran (molecular weight, $75 \mathrm{KDa}$ ) was mechanically crushed into powder. Meanwhile, succinic anhydride was added to $\varepsilon$-poly (L-lysine) solution (10 w/w \%) (PL solution). The PL solution was dyed with brilliant blue FCF (50 ppm) (Wako Pure Chemical Industries, Ltd, Osaka, Japan) for visualization in vivo; $1 \mathrm{~mL}$ of PL solution was stored in each cylinder of a dual-syringe device at $4^{\circ} \mathrm{C}$ until use. For gel formation, the aldehyde dextran and PL solution were mixed at $25^{\circ} \mathrm{C}$. The gel formation started within 10 seconds and was complete within 60 seconds of the time of mixture.

\section{Preparation of Rabbit Pericardiectomy Model}

A total of 39 Japanese white rabbits weighing 3.2 to $4.0 \mathrm{~kg}$ were used. General anesthesia was induced by intravenous injection of ketamine hydrochloride $(5 \mathrm{mg} / \mathrm{kg})$ and sodium thiopental $(15 \mathrm{mg} / \mathrm{kg})$. Rabbits were immediately intubated and connected to mechanical ventilation (tidal volume, $10 \mathrm{~mL} / \mathrm{kg}$; respiratory rate, $30 \mathrm{cycles} / \mathrm{min}$ ). Anesthesia was maintained with isoflurane $(1.0 \%-2.5 \%)$. Intravenous antibiotics $(100 \mathrm{mg} / \mathrm{kg}$ of cefazolin) were administered 30 minutes before the skin incision. The chest hair was shaved, and the skin was disinfected with iodine-alcohol solution. Each rabbit was placed in the supine position, and a midline skin incision was made from just below the suprasternal notch to the xiphoid process. A median sternotomy was performed with surgical scissors while lifting the sternum; care was taken not to injure the pericardium or pleura. A neonate sternal retractor was carefully applied, and the visceral pleura and the pericardium were resected as much as possible. The pericardium between the bilateral phrenic nerves was resected as much as possible (pericardiectomy). In group $1(\mathrm{n}=12)$, the pericardial defect was left as a control. In group $2(\mathrm{n}=12)$, the excised pericardium was substituted with a 0.1-mm-thick ePTFE membrane, which was partly fixed to the edge of the pericardium with 6-0 polypropylene stitches, allowing the fluid not to pool at the retrosternal space. In group $3(n=15)$, the biodegradable glue was applied to the site of the excised pericardium, that is, the epicardium of the right ventricle was coated with the glue. The sternum was closed with 6 interrupted 3-0 polyglactin sutures. The muscle and skin were closed by interrupted 5-0 polypropylene sutures. The inhalation of isoflurane was terminated, and the rabbits were gently recovered with ventilatory support. Intravenous antibiotics $(100 \mathrm{mg} / \mathrm{kg}$ of cephazolin) were administered again.

\section{Macroscopic Evaluation for Retrosternal Adhesion}

Resternotomy was performed 4 weeks after the initial operation. The resternotomy was carefully performed with surgical scissors. Two independent researchers evaluated the macroscopic adhesion in the retrosternal space ( $\mathrm{n}=6$ for each group; midterm evaluation). For the evaluation, the adhesion score established by Heydorn and colleagues ${ }^{19}$ was used. In brief, the degree of adhesion was quantitatively classified from 0 to 4 with modification as follows: $0=$ no adhesion; $1=$ trivial adhesion that could be readily separated by finger dissection; $2=$ mild adhesion requiring blunt dissection; 3 = severe adhesion requiring sharp dissection; and $4=$ undissectable (bleeding occurred from the heart).

\section{Microscopic Evaluation}

The rabbits were killed 4 weeks after the initial operation. For microscopic evaluation, the en bloc thorax, including the sternum, entire heart, and bilateral lungs, was removed through a midline skin incision $(n=6$ for each group; midterm evaluation). The same procedure was conducted 12 weeks after the initial operation for some of the rabbits in group 3 , the biodegradable glue group $(\mathrm{n}=3$; long-term evaluation).

For histologic examination, the samples were divided along the midline and fixed in $10 \%$ formaldehyde, embedded in paraffin, and sliced. The sections were subjected to Masson trichrome stain for the assessment of fibrous reaction. The fibrotic area was automatically detected with a computer-based method. Three random high-magnification $(\times 200)$ views for a Masson trichrome stain section (corresponding to 1 rabbit) were subjected to automatic detection of green fibrotic area using BZ-9000 Software (Keyence Corp, Osaka, Japan), and the fibrotic area ratio was calculated as the average of the values of 3 views.

For the evaluation of biocompatibility of the pericardial substitute, we counted the number of macrophages in the retrosternal space for 5 fields with a magnification of $\times 400$ for each rabbit sample. We used mouse monoclonal anti-rabbit macrophage antibody RAM11 (Dako, Glostrup, Denmark; code No. M0633) for the immunostaining of macrophages.

\section{Statistics}

We used SPSS II for Windows (v 11.0.1J; SPSS Inc, Chicago, Ill) as the statistical software. The results were described as the mean \pm standard deviation. Comparisons among the 3 groups were conducted by 1-way analysis of variance with Tukey's test as the post hoc.

\section{RESULTS}

\section{Effect of the Biodegradable Glue on Retrosternal Adhesion}

Four weeks after the initial operation (midterm), the macroscopic adhesion in group 2 (ePTFE) and group 3 (Lydex, the biodegradable glue) was attenuated compared with that 


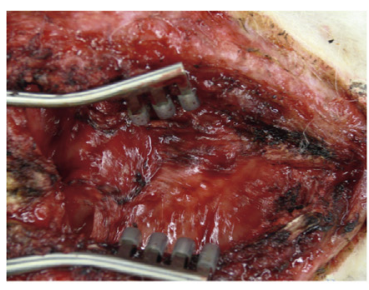

A Group 1 (Control)

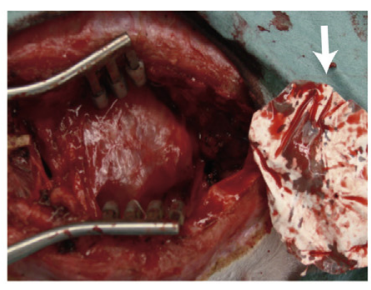

Group 2 (ePTFE)

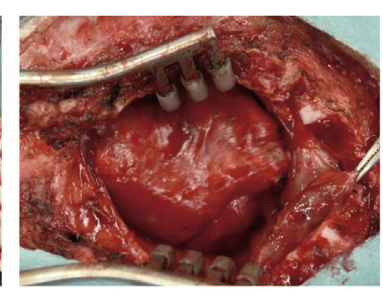

Group 3 (Lydex)

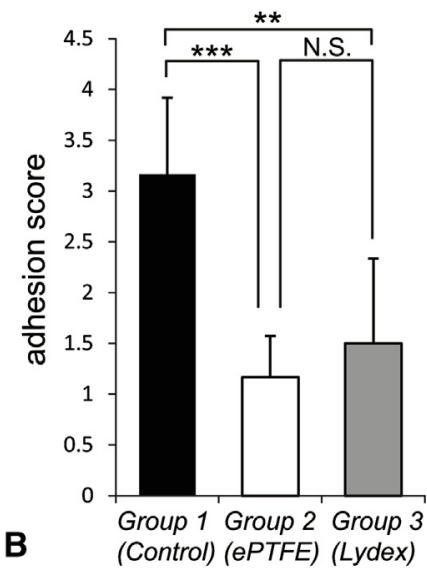

FIGURE 1. The effect of the biodegradable glue on retrosternal adhesion. A, Representative macroscopic view at the evaluation of retrosternal adhesion 4 weeks after the initial operation (midterm). Note that group 1 (control; left) showed more severe adhesion compared with group 2 (ePTFE; middle) and group 3 (Lydex; the biodegradable glue; right). Arrow in the middle indicates excised ePTFE membrane. B, Adhesion score ( $\mathrm{n}=6$ for each group). $* * P<.01$. *** $P<.001 . e P T F E$, Expanded polytetrafluoroethylene; N.S., not significant.

in group 1 (normal control) (Figure 1, A; the representative macroscopic views recorded before dissection of retrosternal adhesion). The adhesion scores were $3.17 \pm 0.75$ in group $1,1.17 \pm 0.41$ in group 2, and $1.50 \pm 0.84$ in group 3 (Figure 1, B). The retrosternal adhesion score in group 3 was significantly lower than in group $1(P=.0022)$. However, there was no significant difference between scores for groups 2 and $3(P=.69)$. These results indicate that the ePTFE membrane and application of the biodegradable glue significantly reduced the retrosternal adhesion to the same extent 4 weeks after the pericardiectomy.

\section{Effect of the Biodegradable Glue on Fibrosis}

Representative sections of Masson trichrome staining of each group at 4 weeks after the initial operation (midterm) are shown in Figure 2, A. Severe fibrous adhesion was observed between the sternum and the myocardium, and no pericardial space was observed in group 1 . In group 2, although the retrosternal fibrosis was denser than that in group 1, fibrosis-free space was observed on both sides of the ePTFE membrane. In group 3, retrosternal fibrosis was diminished compared with groups 1 and 2 , and pericardial space was observed. However, the glue remained on the epicardium, and granulomatous tissue formation was observed around the residual glue. The fibrotic area ratio was $50.3 \% \pm 12.1 \%$ in group $1,64.5 \% \pm 11.1 \%$ in group 2 , and $22.0 \% \pm 8.1 \%$ in group 3 (Figure 2, B). In group 2, the fibrotic area ratio was higher than in group 1 but not statistically significant $(P=.08)$. However, the fibrous reaction score in group 3 was significantly lower than in group $1(P<.001)$ or group $2(P<.001)$. This indicates that the biodegradable glue attenuates the progression of fibrosis after the initial operation, whereas ePTFE membrane implantation shows a tendency for promotion of fibrosis. In all observed rabbits 12 weeks after the glue implantation (long-term, $\mathrm{n}=3$ ), the residual glue and granulomatous tissue observed with midterm section disappeared, and no progression of adhesion or fibrosis was observed (Figure 2, C). This result indicates that the glue degrades with time and that implantation does not promote adhesion because of chronic inflammation.

\section{Biocompatibility of the Biodegradable Glue}

Representative sections of RAM11 staining of each group at 4 weeks after the initial operation (midterm) are shown in Figure 3, A. A prominent infiltration of macrophages was observed in the section of group 2 compared with the other groups. The macrophage counts were $31.0 \pm 13.7$ in group $1,85.7 \pm 27.7$ in group 2, and $30.0 \pm 6.8$ in group 3 (Figure $3, B$ ). The number of 

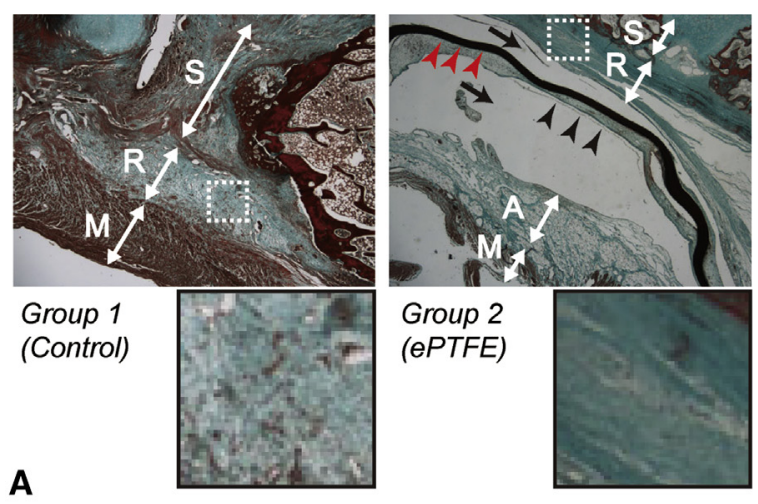

Group 2 (ePTFE)

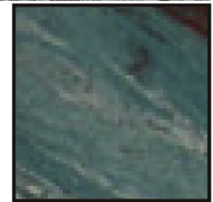

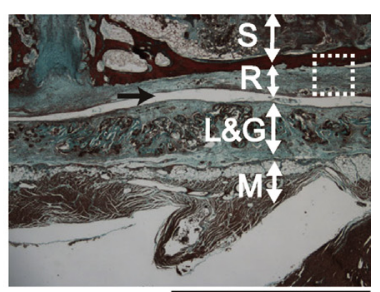

Group 3 (Lydex)
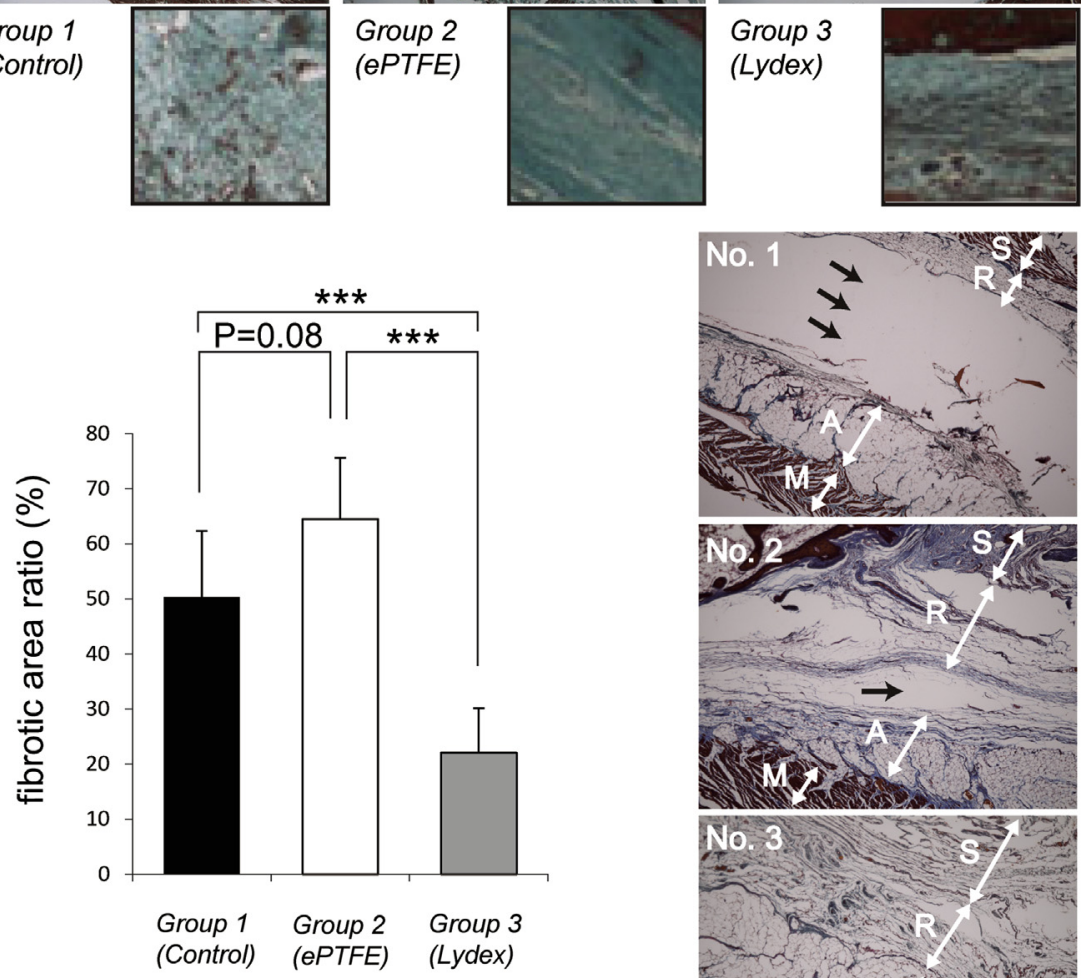

B

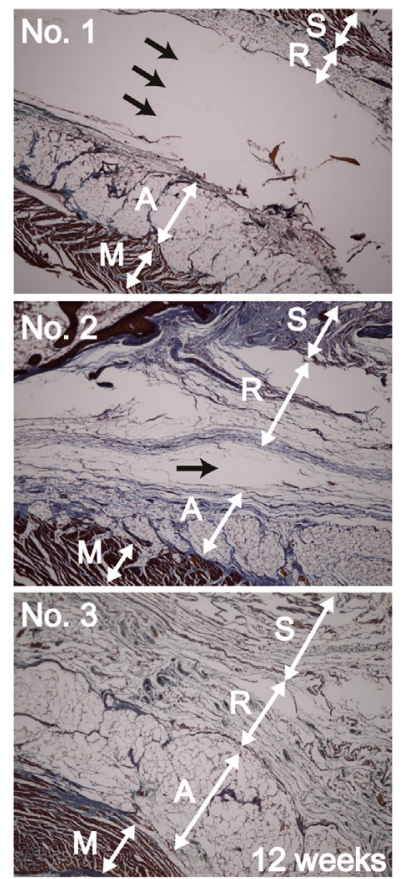

FIGURE 2. The effect of the biodegradable glue on fibrosis. A, Representative Masson trichrome staining (4 weeks after initial operation; midterm) (magnification: $\times 100$ ). Magnification of retrosternal region (broken square) is shown (lower right). Green area indicates fibrotic tissue. Arrows in the middle and to the right indicate pericardial cavity. Red arrowheads in the middle indicate ePTFE membrane. Black arrowheads in the middle indicate granulation tissue around the ePTFE membrane. B, Fibrotic area ratio ( $\mathrm{n}=6$ for each group). $* * * P<.001$. C, Representative Masson trichrome staining in group 3 (Lydex, the biodegradable glue). Sections of all rabbits included in this long-term evaluation (12 weeks after initial operation) are shown ( $\mathrm{n}=3$; from No. 1-3). Arrow indicates pericardial cavity. $S$, Sternum; $R$, retrosternal space; $M$, myocardium; $A$, adipose tissue; $L \& G$, Lydex and granulation tissue; $e P T F E$, expanded polytetrafluoroethylene.

macrophages in group 2 was significantly higher than in group $1(P=.00035)$. However, the number of macrophages in group 3 was significantly lower than in group 2 $(P=.00030)$. There was no significant difference between counts for groups 1 and 3 . These results indicate that the biodegradable glue causes little foreign-body reaction on retrosternal application and possesses excellent biocompatibility compared with ePTFE membrane.

\section{DISCUSSION}

In cardiac reoperations, dense retrosternal adhesions between the sternum and the heart can induce fatal bleeding during resternotomy, as well as right ventricular dysfunction or decreased coronary artery bypass graft patency. Consequently, it is said that the postoperative retrosternal adhesion increases total surgical risk during reoperation. ${ }^{2,3}$ Therefore, efforts made to prevent subsequent adhesion during an initial operation may lead to an improvement in surgical outcome.

Postoperative adhesion, which is formed after initial operation, is scar formation as the end stage of the inflammatory response. It is also known as the process of wound healing. Wound healing is classified into 3 phases: inflammation, proliferation, and maturation. ${ }^{20}$ The initial injury 


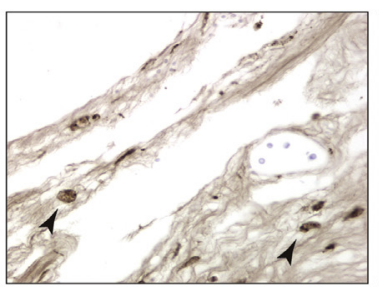

A Group 1 (Control)

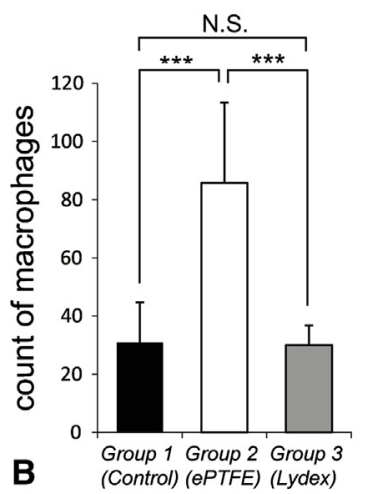

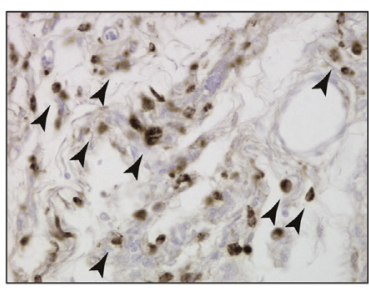

Group 2 (ePTFE)

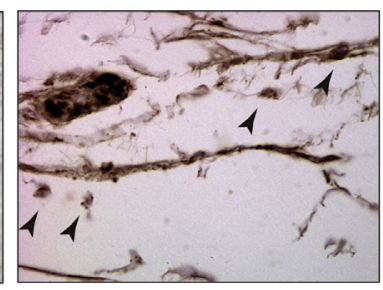

Group 3 (Lydex)

FIGURE 3. The biocompatibility of the biodegradable glue. A, Representative RAM 11 staining for macrophages (4 weeks after initial operation; midterm) (magnification: $\times 400$ ). Brown spots indicate macrophages (arrowheads). B, Count of macrophages $(\mathrm{n}=6$ for each group; each count is the average count of 5 random fields with $\times 400$ magnification macroscopic view). $* * * P<.001$. ePTFE, Expanded polytetrafluoroethylene; N.S., not significant.

causes platelet adhesion and aggregation and the formation of clots in the surface of the wound, leading to inflammation. In the proliferative phase (within the initial 24-72 hours from injury), formation of granulation tissue takes place because of proliferation and migration of connective tissue cells, fibroblasts, or vascular endothelial cells. Macrophages are the main cell components involved with tissue repair as the main source for multiple growth factors, which trigger proliferation of fibroblasts and control migration of fibroblasts to the site of injury. In the maturation phase, the granulation tissue scaffolding is converted into avascular scar, composed of spindle-shaped fibroblasts, dense collagen, and fragments of elastic tissue. By the end of the first month, the scar is made up of acellular, fibrotic connective tissue devoid of inflammatory infiltration and induces local adhesion. For the purpose of attenuating dense retrosternal adhesions, it is essential to suppress the progression of the vicious inflammatory response described earlier.

In this study, we demonstrated that the biodegradable glue, a new material derived from food additives, significantly reduced postoperative adhesion. In a rabbit model of pericardiectomy, application of the glue prevented retrosternal adhesion as well as ePTFE membrane. Furthermore, histologic studies revealed that application of the glue suppressed fibrosis and foreign-body reaction after the surgical procedure.

The preventive effect of the biodegradable glue on adhesion was comparable to that of ePTFE membrane. ePTFE membrane is the most commonly used synthetic pericardial substitute; it serves as a barrier between sternum and epicardium and makes re-sternotomy safe. Thus, our results suggest that the application of the glue may profit cardiac surgeons in the clinical setting. One of the disadvantages of ePTFE membrane is severe inflammatory reaction and diffuse fibrosis around the membrane, as has been observed in our studies. In the present study, fibrosis and foreignbody reaction after application of the biodegradable glue were almost comparable to those of the control. These results suggest that application of the glue inhibits the wound-healing process. The mechanism of the glue in preventing postoperative adhesion should be examined further.

The present study also demonstrated the excellent biocompatibility of the biodegradable glue. After implantation, macrophages recognize materials as foreign substances and then activate and trigger phagocytosis for the substances. When implanted foreign substances such as sutures or artificial materials (eg, ePTFE, the biodegradable glue studied) are large enough that phagocytosis by a single macrophage is precluded, the macrophages remain in the periphery of the substances. Their presence indicates the continuation of chronic inflammation, which is distinct from granulomatous inflammation. Therefore, the degree of chronic inflammation in a foreign-body reaction is proportional to the number of macrophages in the periphery of the substance. Conversely, the number of macrophages is inversely proportional to the degree of biocompatibility of the implanted foreign substances. In the present study, the number of macrophages in the biodegradable glue group was significantly lower than in the ePTFE group and almost equal to the 
number in the control group. Furthermore, in any rabbit with the implanted glue (4-week and 12-week models), there was no sign of constrictive pericarditis, a pathologic consequence of chronic inflammation. These results indicate that the glue causes the least chronic inflammatory reaction and possesses excellent biocompatibility, with a suppressed foreign-body reaction after implantation. Furthermore, it is confirmed in the present study that the glue does not remain 12 weeks after implantation. Another recent study showed that the biodegradable glue was fully absorbed after 28 days of local administration in the pleural cavity of rats. ${ }^{21}$ Thus, it can be concluded that the glue has excellent biocompatible and biodegradable properties.

In regard to the safety of the biodegradable glue for clinical application, it is likely to be safe for the human body with regard to cell toxicity, as shown by Nakajima and colleagues. ${ }^{22}$ Furthermore, because the glue is made of food additives, there must be a limited risk for viral infections, such as hepatitis virus or human immunodeficiency virus, compared with the risk from materials derived from human plasma or other animal-derived products.

Another advantage of the biodegradable glue is the low cost. The main materials of the glue are derived from food additives and available at a lower cost compared with human or animal blood-derived materials, which may be favorable for worldwide use of this material.

For clinical application of the biodegradable glue, we need further experiments using large-scale animals (eg, porcine model) that would be more similar to the clinical setting compared with the rabbit model used in the present study. In the preclinical studies, the safety of the local application of this novel material must be confirmed in addition to the preventive effect of retrosternal adhesion.

\section{Study Limitations}

As described earlier, there are many usable materials for the prevention of postoperative adhesion. The use of CoSeal (Baxter Healthcare, Deerfield, Ill) has shown an excellent outcome for the prevention of retrosternal adhesion in randomized animal studies ${ }^{23}$ or observational studies in pediatric cardiac surgery. ${ }^{24}$ More recently, CorMatrix (CorMatrix Cardiovascular, Atlanta, Ga) has been applied for pericardial reconstruction as extracellular matrices for tissue regeneration. $^{25}$ However, in this article, we merely compared the properties of the biodegradable glue and ePTFE membrane. Whereas the safety of the glue with regard to the low probability of viral infection is a potent and specific advantage of this material, there might be other materials that have the same excellent biocompatibility and protective effect for fibrosis formation as the glue. Further examinations are needed to determine whether the biodegradable glue holds more advantages for the prevention of retrosternal adhesion compared with other new materials, such as CoSeal or CorMatrix.
We suppose that the use of the biodegradable glue has more limited possibilities for surgical wound infection because of its biodegradability, and it is advantageous compared with the use of ePTFE membrane. However, we have not closely assessed local or systemic infection in this study. Although macroscopically apparent infection was not observed in any rabbit included in this study, closer examination for bacterial or viral infections should have been conducted to demonstrate superior infection resistance of the biodegradable glue compared with ePTFE membrane.

\section{CONCLUSIONS}

Lydex, a novel bioabsorbable glue derived from food additives, is an excellent material for prevention of retrosternal adhesion after surgical procedures and does not increase fibrosis or macrophage infiltration in the retrosternal area because of its excellent biocompatibility. The biodegradable glue may be an ideal material to prevent postoperative retrosternal adhesion.

\section{References}

1. Sakata R, Fujii Y, Kuwano H. Thoracic and cardiovascular surgery in Japan during 2009. Gen Thorac Cardiovasc Surg. 2011;59:636-67.

2. Yau TM, Borger MA, Weisel RD, Ivanov J. The changing pattern of reoperative coronary surgery: trends in 1230 consecutive reoperations. J Thorac Cardiovasc Surg. 2000;120:156-63.

3. Loop FD. Catastrophic hemorrhage during sternal reentry. Ann Thorac Surg. 1984;37:271-2.

4. Revuelta JM, Rinaldi RG. Expanded polytetrafluoroethylene surgical membrane for pericardial closure. J Thorac Cardiovasc Surg. 1985;89:451-5.

5. Laks H, Hammond G. Use of silicone rubber as a pericardial substitute to facilitate reoperation in cardiac surgery. J Thorac Cardiovasc Surg. 1981;82:88-92.

6. Bunton RW, Xabregas AA. Pericardial closure after cardiac operations. J Thorac Cardiovasc Surg. 1990;100:99-107.

7. Mathisen SR, Sauvage LR. Prevention of retrosternal adhesions after pericardiotomy. J Thorac Cardiovasc Surg. 1986;92:92-8.

8. Okuyama N, Wang CY, Rose EA, et al. Reduction of retrosternal and pericardial adhesions with rapidly resorbable polymer films. Ann Thorac Surg. 1999;68:913-8.

9. Goëau-Brissonnière $\mathrm{O}$, Leport $\mathrm{C}$, Guidoin $\mathrm{R}$, Lebrault $\mathrm{C}$, Péchère $\mathrm{JC}$, Bacourt $\mathrm{F}$. Experimental colonization of an expanded polytetrafluoroethylene vascular graft with Staphylococcus aureus: a quantitative and morphologic study. J Vasc Surg. 1987;5:743-8

10. Bel A, Ricci M, Piquet J, Bruneval P, Perier MC, Gagnieu C, et al. Prevention of postcardiopulmonary bypass pericardial adhesions by a new resorbable collagen membrane. Interact Cardiovasc Thorac Surg. 2012;14:469-73.

11. Lodge AJ, Wells WJ, Backer CL, O'Brien JE Jr, Austin EH, Bacha EA, et al. A novel bioresorbable film reduces postoperative adhesions after infant cardiac surgery. Ann Thorac Surg. 2008;86:614-21.

12. Naito Y, Shin'oka T, Hibino N, Matsumura G, Kurosawa H. A novel method to reduce pericardial adhesion: a combination technique with hyaluronic acid biocompatible membrane. J Thorac Cardiovasc Surg. 2008;135:850-6.

13. Yoshioka I, Saiki Y, Sakuma K, Iguchi A, Moriya T, Ikada Y, et al. Bioabsorbable gelatin sheets latticed with polyglycolic acid can eliminate pericardial adhesion. Ann Thorac Surg. 2007;84:864-70.

14. Araki M, Tao H, Nakajima N, Sugai H, Sato T, Hyon SH, et al. Development of new biodegradable hydrogel glue for preventing alveolar air leakage. $J$ Thorac Cardiovasc Surg. 2007;134:1241-8.

15. Hiraki J, Ichikawa T, Ninomiya S, Seki H, Uohama K, Seki H, et al. Use of ADME studies to confirm the safety of epsilon-polylysine as a preservative in food. Regul Toxicol Pharmacol. 2003;37:328-40.

16. Morishima M, Marui A, Yanagi S, Nomura T, Nakajima N, Hyon SH, et al. Sustained release of vancomycin from a new biodegradable glue to prevent methicillin-resistant Staphylococcus aureus graft infection. Interact Cardiovasc Thorac Surg. 2010;11:52-5. 
17. Takeda T, Shimamoto T, Marui A, Saito N, Uehara K, Minakata K, et al. Topical application of a biodegradable disc with amiodarone for atrial fibrillation. Ann Thorac Surg. 2011;91:734-9.

18. Masumoto H, Sakata R. Cardiovascular surgery for realization of regenerative medicine. Gen Thorac Cardiovasc Surg. 2012;60:744-55.

19. Heydorn WH, Daniel JS, Wade CE. A new look at pericardial substitutes. J Thorac Cardiovasc Surg. 1987;94:291-6.

20. Kumar V, Abbas AK, Fausto N, Aster J. Acute and chronic inflammation. In: Smeraldi C, Lesciera P, eds. Robbins \& Cotran Pathologic Basis of Disease. 8th ed. Philadelphia, PA: Elsevier; 2010:43-77.

21. Takagi K, Tsuchiya T, Araki M, Yamasaki N, Nagayasu Y, Hyon SH, et al. Novel biodegradable powder for preventing postoperative pleural adhesion. J Surg Res. 2013;179:e13-9.
22. Nakajima N, Sugai H, Tsutsumi S, Hyon SH. Self-degradable bioadhesive. Key Eng Mater. 2007;342-3:713-6.

23. Marc Hendrikx M, Mees U, Hill AC, Egbert B, Coker GT, Estridge TD. Evaluation of a novel synthetic sealant for inhibition of cardiac adhesions and clinical experience in cardiac surgery procedures. Heart Surg Forum. 2001;4: 204-10.

24. Pace Napoleone C, Valori A, Crupi G, Ocello S, Vouché P, et al. An observational study of CoSeal for the prevention of adhesions in pediatric cardiac surgery. Interact Cardiovasc Thorac Surg. 2009;9:978-82.

25. Boyd WD, Johnson WE III, Sultan PK, Deering TF, Matheny RG. Pericardial reconstruction using an extracellular matrix implant correlates with reduced risk of postoperative atrial fibrillation in coronary artery bypass surgery patients. Heart Surg Forum. 2010;13:E311-6. 